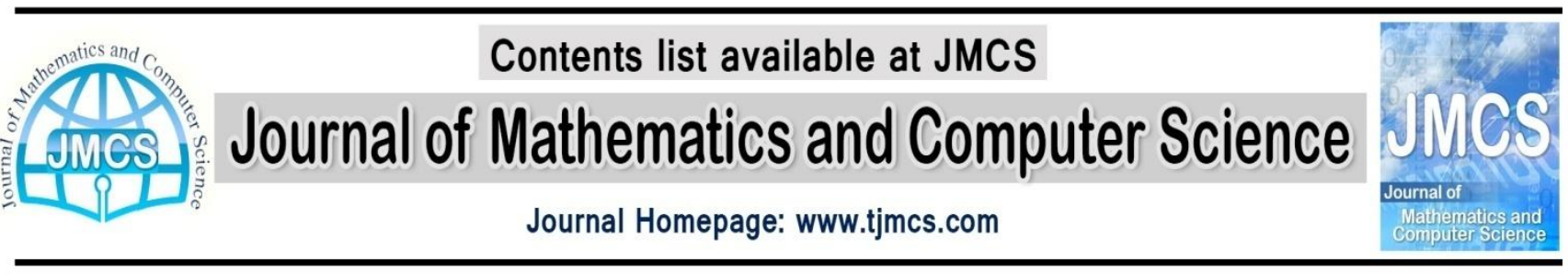

\title{
On a Semi-Symmetric Non-Metric Connection in an Indefinite Para Sasakian Manifold
}

\author{
S.K. Pandey ${ }^{1}$, G.Pandey ${ }^{2}$, K.Tiwari ${ }^{3}$ and R.N. Singh ${ }^{4}$ \\ Department of Mathematical Sciences, A.P.S. University, Rewa(M.P.),486003, India. \\ 1. shravan.math@gmail.com, 2. math.giteshwari@gmail.com, 3. kirantiwari@yahoo.com, \\ 4. rnsinghmp@ rediffmail.com
}

Article history:

Received June 2014

Accepted July 2014

Available online August 2014

\begin{abstract}
The object of the present paper is to study a semi-symmetric non-metric connection in an indefinite para Sasakian manifold. In this paper, we obtain the relation between the semi-symmetric non-metric connection and Levi-Civita connection in an indefinite para Sasakian manifold. Also, the Nijenhuis tensor, curvature tensor and projective curvature tensor of semi-symmetric non-metric connection in an indefinite para Sasakian manifold have been studied.
\end{abstract}

AMS Mathematics Subject Classification : 53C15, $53 \mathrm{C} 25$.

Key Words: Indefinite para Sasakian manifold, semi-symmetric non-metric connection, Nijenhuis tensor, projective curvature tensor.

\section{Introduction}

The idea of semi-symmetric connection was introduced by Friedmann and Schouten [13] in 1924. A linear connection $\bar{\nabla}$ on a Riemannian manifold $\left(M^{n}, \mathrm{~g}\right)$ is called semi-symmetric, if its torsion tensor $\bar{T}$ satisfies

$$
\bar{T}(X, Y)=\eta(Y) X-\eta(X) Y,
$$

where $\eta$ is a non-zero 1-form associated with a vector field $\xi$ defined by

$$
\eta(X)=g(X, \xi)
$$


In 1930, Bartolotti [4] gave geometrical meaning to such a connection. In 1932, Hayden [14] defined and studied semi-symmetric metric connection. In 1970, Yano [22] started the systematic study of semisymmetric metric connection and this was further developed by various authors such as Amur and Pujar [2], Chaki and Chaki [6], Chaki and Konar [7], De [8], De and De [9], De and Ghosh [10], Imai [15], Mishra and Pandey [17], Prvanovic [18], Singh and Pandey [20], Yano and Imai ([23], [24]) and several other geometers.

In 1975, Prvanovic [18] introduced the concept of semi-symmetric non-metric connection with the name pseudo metric which was further studied by Andonic [3]. The study of semi-symmetric non-metric connection is much ancient than the nomenclature 'non-metric' was introduced.

In 1992, Agashe and Chafle [1] introduced a semi-symmetric connection $\bar{\nabla}$ satisfying $\bar{\nabla}_{X} g \neq 0$ and called such a connection as semi-symmetric non-metric connection. They gave the relation between the curvature tensors of the manifold with respect to the semi-symmetric non-metric connection and the Riemannian connection. They also proved that the projective curvature tensors of the manifold with respect to these connections are equal to each other.

The study of manifolds with indefinite metric is of interest from the stand point of physics and relativity. Manifolds with indefinite metrics have been studied by several authors. In 1993, Bejancu and Duggal [5] introduced the concept of $(\varepsilon)$-Sasakian manifolds and Xufeng and Xiaoli [20] established that these manifolds are real hypersurfaces of indefinite Kahlerian manifold. Recently De and Sarkar [11] introduced $(\varepsilon)$-Kenmotsu manifolds and studied conformally flat, weyl semisymmetric, $\phi$-recurrent $(\varepsilon)$ Kenmotsu manifolds. Motivated by the above studies, in the present paper, we have studied semisymmetric non-metric connection in an $(\varepsilon)$-para Sasakian manifold. The present paper is organized as follows-

In section 2, some preliminary results regarding indefinite Sasakian manifolds are recalled. Section 3 deals with semi-symmetric non-metric connection in $(\varepsilon)$-para Sasakian manifold. Section 4 is devoted to the study of Nijenhuis tensor of semi-symmetric non metric connection. In section 5 , curvature tensor of semi-symmetric non-metric connection have been studied. The last section deals with projective curvature tensor of semi-symmetric non-metric connection in $M^{n}$.

\section{$2(\varepsilon)$-Para Sasakian Manifolds}

Let $M^{n}$ be an almost para contact manifold equipped with an almost paracontact structure $(\phi, \xi, \eta)$ consisting of a tensor field $\phi$ of type $(1,1)$, a vector field $\xi$ and a one form $\eta$ satisfying

$$
\begin{gathered}
\phi^{2}=I-\eta \otimes \xi, \\
\eta(\xi)=1,
\end{gathered}
$$




$$
\phi(\xi)=0
$$

and

$$
\eta \circ \phi=0 .
$$

Let $M^{n}$ be an $\mathrm{n}$-dimensional almost paracontact manifold and $\mathrm{g}$ be semi-Riemannian metric with index $(\mathrm{g})=v$ such that

$$
g(\phi X, \phi Y)=g(X, Y)-\varepsilon \eta(X) \eta(Y),
$$

where $\varepsilon= \pm 1$. In this case, $M^{n}$ is called an $(\varepsilon)$ - almost paracontact metric manifold equipped with an $(\varepsilon)$ - almost paracontact structure $(\phi, \xi, \eta, g)$ [21]. In particular, if index(g) $=1$, then an $(\varepsilon)$ - almost paracontact metric manifold will be called a Lorentzian almost paracontact manifold. If in case, the metric is positive definite, then an $(\varepsilon)$ - almost paracontact metric manifold is the usual almost paracontact metric manifold [19].

In view of equations (4), (5) and (7), we have

$$
g(X, \phi Y)=g(\phi X, Y)
$$

and

$$
g(X, \xi)=\varepsilon \eta(X)
$$

for all $X, Y \in T M^{n}$. From equation (9), it follows that

$$
g(\xi, \xi)=\varepsilon,
$$

i.e. the structure vector field $\xi$ is never light like. An $(\varepsilon)$ - almost paracontact metric manifold (resp., a Lorentzian almost paracontact manifold $\left(M^{n}, \phi, \xi, \eta, g, \varepsilon\right)$ is said to be space like $(\varepsilon)$ - almost paracontact metric manifold (respectively a space like Lorentzian almost paracontact manifold), if $\varepsilon=1$ and $M^{n}$ is said to be a time like $(\varepsilon)$ - almost paracontact metric manifold (respectively a Lorentzian almost paracontact manifold), if $\varepsilon=-1$.

An $(\varepsilon)$ - almost paracontact metric structure is called an $(\varepsilon)$ - para Sasakian structure if

$$
\left(\nabla_{X} \phi\right)(Y)=-g(\phi X, \phi Y) \xi-\xi \eta(Y) \phi^{2} X, \quad X, Y \in T M^{n},
$$

where $\nabla$ is the Levi-Civita connection. A manifold $M^{n}$ endowed with an $(\varepsilon)$-para Sasakian structure is called an $(\varepsilon)$-para Sasakian manifold. For $\varepsilon=1$ and $\mathrm{g}$ Riemannian, $M^{n}$ is the usual para Sasakian manifold [19]. For $\varepsilon=-1$, g Lorentzian and $\xi$ replaced by- $\xi, M^{n}$ becomes a Lorentzian para Sasakian manifold [12].

In an $(\varepsilon)$-para Sasakian manifold, we have

$$
\nabla_{X} \xi=\varepsilon \phi,
$$




$$
\Omega(X, Y)=\varepsilon g(\phi X, Y)=\left(\nabla_{X} \eta\right)(Y),
$$

for all $X, Y \in T M^{n}$, where $\Omega$ is the fundamental 2-from.

In an $(\varepsilon)$ - almost para Sasakian manifold $M^{n}$, the following relations holds.

$$
\begin{gathered}
R(\xi, X) Y=-\varepsilon g(X, Y) \xi+\varepsilon \eta(Y) X, \\
R(X, Y) \xi=-\varepsilon \eta(Y) X+\varepsilon \eta(X) Y, \\
\eta(R(X, Y) Z)=-\varepsilon[g(Y, Z) \eta(X)-g(X, Z) \eta(Y)] .
\end{gathered}
$$

In an n-dimensional $(\varepsilon)$ - para Sasakian manifold $M^{n}$, the Ricci tensor $\mathrm{S}$ satisfies

$$
S(\phi Y, \phi Z)=S(Y, Z)+(n-1) \eta(Y) \eta(Z),
$$

for all $\mathrm{X}, \mathrm{Y} \in T M^{n}$. By virtue of above equation, we have

$$
S(\phi Y, Z)=S(Y, \phi Z)
$$

and

$$
S(Y, \xi)=-(n-1) \eta(Y) .
$$

Example 1 Let $R^{3}$ be the 3-dimensional real number space with a co-ordinate system $(x, y, z)$ we define

$$
\begin{gathered}
\eta=d z-y d x, \quad \xi=\frac{\partial}{\partial z}, \\
\varphi\left(\frac{\partial}{\partial x}\right)=-\frac{\partial}{\partial x}-y \frac{\partial}{\partial z}, \quad \varphi\left(\frac{\partial}{\partial y}\right)=-\frac{\partial}{\partial y}, \quad \varphi\left(\frac{\partial}{\partial z}\right)=0, \\
g_{1}=(d x)^{2}+(d y)^{2}-\eta \otimes \eta, \\
g_{2}=(d x)^{2}+(d y)^{2}+(d z)^{2}-y(d x \otimes d z+d z \otimes d x), \\
g_{3}=-(d x)^{2}+(d y)^{2}+(d z)^{2}-y(d x \otimes d z+d z \otimes d x) .
\end{gathered}
$$

then the $\operatorname{set}(\varphi, \xi, \eta)$ is an almost paracontact structure in $R^{3}$. The set $\left(\varphi, \xi, \eta, g_{1}\right)$ is a timelike Lorentzain almost paracontact structure. Moreover, the trajectories of the timelike structure vector $\xi$ 
are geodesics. The set $\left(\varphi, \xi, \eta, g_{2}\right)$ is a spacelike Lorentzian almost paracontact structure. The set $\left(\varphi, \xi, \eta, g_{3}\right)$ is a spacelike $(\varepsilon)$-almost paracontact metric structure $\left(\varphi, \xi, \eta, g_{3}, \varepsilon\right)$ with index $\left(g_{3}\right)=2$.

\section{Semi-Symmetric Non-Metric Connection}

Let $\bar{\nabla}$ be a linear connection and $\nabla$ be a Levi-Civita connection of an $(\varepsilon)$ - para Sasakian manifold $M^{n}$ such that

$$
\bar{\nabla}_{X} Y=\nabla_{X} Y+H(X, Y),
$$

where $\mathrm{H}$ is tensor of type $(1,2)$. For $\bar{\nabla}$ to be a semi-symmetric non-metric connection in $M^{n}$, we have

$$
H(X, Y)=\frac{1}{2}\left[\bar{T}(X, Y)+\bar{T}^{*}(X, Y)+\bar{T}^{*}(Y, X)\right]+g(X, Y) \xi,
$$

where $\bar{T}^{*}$ is a tensor of type $(1,2)$ defined on $M^{n}$ as

$$
g(\bar{T}(Z, X), Y)=g\left(\bar{T}^{*}(X, Y), Z\right) .
$$

By virtue of equation (1), equation (22) takes the form

$$
\bar{T}^{*}(X, Y)=\eta(X) Y-g(X, Y) \xi .
$$

Using equations (1) and (23) in equation (21), we get

$$
H(X, Y)=\eta(Y) X .
$$

Hence in view of equations (20) and (24), a Semi-Symmetric connection on an $(\varepsilon)$ - para Sasakian manifold $M^{n}$ is given by

$$
\bar{\nabla}_{X} Y=\nabla_{X} Y+\eta(Y) X .
$$

Conversely, the torsion tensor $\bar{T}$ of the connection $\bar{\nabla}$ defined in equation (25) is given by

$$
\begin{aligned}
\bar{T}(X, Y) & =\bar{\nabla}_{X} Y-\bar{\nabla}_{Y} X-[X, Y] \\
& =\nabla_{X} Y+\eta(Y) X-\nabla_{X} Y-\eta(X) Y-[X, Y],
\end{aligned}
$$

i.e.

$$
\bar{T}(X, Y)=\eta(Y) X-\eta(X) Y .
$$

The above equation shows that the connection $\bar{\nabla}$ is a semi-symmetric connection. Also, we have

$$
\left(\bar{\nabla}_{X} g\right)(Y, Z)=\bar{\nabla}_{X} g(Y, Z)-g\left(\bar{\nabla}_{X} Y, Z\right)-g\left(Y, \bar{\nabla}_{X} Z\right),
$$

i.e

$$
\left(\bar{\nabla}_{X} g\right)(Y, Z)=-\eta(Y) g(X, Z)-\eta(Z) g(X, Y)
$$


In view of equations (26) and (27), we conclude that the connection $\bar{\nabla}$ is a semi-symmetric non-metric connection. Thus equation (25) is the relation between the Levi-Civita connection $\nabla$ and semi-symmetric non-metric connection $\bar{\nabla}$ on an $(\varepsilon)$ - para Sasakian manifold $M^{n}$.

Now, in view of equation (25), we have

$$
\left(\bar{\nabla}_{X} \phi\right)(Y)=\bar{\nabla}_{X} \phi(Y)-\phi\left(\bar{\nabla}_{X} Y\right)
$$

i.e.

$$
\left(\bar{\nabla}_{X} \phi\right)(Y)=\left(\nabla_{X} \phi\right)(Y)-\eta(Y) \phi(X) .
$$

Replacing $X$ and $Y$ by $\bar{X}$ and $\bar{Y}$ respectively, where $\bar{X}=\phi X$ and using equation (6), we get

$$
\left(\bar{\nabla}_{\bar{X}} \phi\right)(\bar{Y})=\left(\nabla_{\bar{X}} \phi\right)(\bar{Y})=-g\left(\phi^{2} X, \phi^{2} Y\right) \xi, \quad X, Y \in T M^{n} .
$$

Thus, we can state as follows -

Theorem 3.1 An n-dimensional ( $\varepsilon$ )- para Sasakian manifold admitting semi-symmetric non-metric connection $\bar{\nabla}$ satisfies equation (29).

Now, we have

$$
\left(\bar{\nabla}_{X} \Omega\right)(Y, Z)=\bar{\nabla}_{X} \Omega(Y, Z)-\Omega\left(\bar{\nabla}_{X} Y, Z\right)-\Omega\left(Y, \bar{\nabla}_{X} Z\right)
$$

In view of equation (25), above equation takes the form

$$
\left(\bar{\nabla}_{X} \Omega\right)(Y, Z)=\left(\nabla_{X} \Omega\right)(Y, Z)-\eta(Y) \Omega(X, Z)-\eta(Z) \Omega(Y, X)
$$

Replacing $Y$ and $Z$ by $\bar{Y}$ and $\bar{Z}$ respectively in above equation and using equation (6), we get

$$
\left(\bar{\nabla}_{X} \Omega\right)(\bar{Y}, \bar{Z})=\left(\bar{\nabla}_{X} \Omega\right)(\bar{Y}, \bar{Z})
$$

Thus we can state as follows

Theorem 3.2 An n-dimensional ( $\varepsilon$ )- para Sasakian manifold admitting semi-symmetric non-metric connection $\bar{\nabla}$ satisfies equation (32).

Now, writing two more equations by the cyclic permutations of $X, Y$ and $Z$ from equation (31), we get

$$
\left(\bar{\nabla}_{Y} \Omega\right)(Z, X)=\left(\nabla_{Y} \Omega\right)(Z, X)-\eta(Z) \Omega(Y, X)-\eta(X) \Omega(Z, Y)
$$

and

$$
\left(\bar{\nabla}_{Z} \Omega\right)(X, Y)=\left(\nabla_{Z} \Omega\right)(X, Y)-\eta(X) \Omega(Z, Y)-\eta(Y) \Omega(X, Z) .
$$


Now $\bar{d} \Omega(X, Y, Z)$ is defined as

$$
\bar{d} \Omega(X, Y, Z)=\left(\bar{\nabla}_{X} \Omega\right)(Y, Z)+\left(\bar{\nabla}_{Y} \Omega\right)(Z, X)+\left(\bar{\nabla}_{Z} \Omega\right)(X, Y) .
$$

By virtue of equations (31), (33) and (34), equation (35) takes the form

$$
\begin{aligned}
\bar{d} \Omega(X, Y, Z) & =d \Omega(X, Y, Z)-2[\Omega(X, Z) \eta(Y) \\
& +\eta(X) \Omega(Y, Z)+\eta(Z) \Omega(X, Y)] .
\end{aligned}
$$

Thus, we conclude that

Theorem 3.3 An n-dimensional ( $\varepsilon$ )- para Sasakian manifold admitting semi-symmetric non-metric connection $\bar{\nabla}$ satisfies equation (36).

Now, from equation (21), we have

$$
\begin{aligned}
{ }^{\prime} H(X, Y, Z) & =\frac{1}{2}\left[{ }^{\prime} \bar{T}(X, Y, Z)+{ }^{\prime} \bar{T}(Z, X, Y)\right. \\
& \left.+{ }^{\prime} \bar{T}(Z, Y, X)\right]+\varepsilon \eta(Z) g(X, Y),
\end{aligned}
$$

where ${ }^{\prime} H(X, Y, Z)=g\left(H(X, Y), Z\right.$ and ${ }^{\prime} \bar{T}(X, Y, Z)=g(\bar{T}(X, Y), Z)$. Interchanging $X$ and $Y$ in above equations, we get

$$
\begin{aligned}
{ }^{\prime} H(Y, X, Z) & =\frac{1}{2}\left[{ }^{\prime} \bar{T}(Y, X, Z)+{ }^{\prime} \bar{T}(Z, X, Y)\right. \\
& \left.+{ }^{\prime} \bar{T}(Z, Y, X)\right]+\varepsilon \eta(Z) g(Y, X) .
\end{aligned}
$$

Now subtracting equation (37) and (38), we get

$$
' \bar{T}(X, Y, Z)-{ }^{\prime} \bar{T}(Y, X, Z)=2\left[{ }^{\prime} H(X, Y, Z)-{ }^{\prime} H(Y, X, Z)\right] .
$$

Thus we can state as follows

Theorem 3.4 On an ( $\varepsilon)$ - para Sasakian manifold with respect to semi-symmetric non-metric connection, we have

$$
' \bar{T}(X, Y, Z)-{ }^{\prime} \bar{T}(Y, X, Z)=2\left[{ }^{\prime} H(X, Y, Z)-{ }^{\prime} H(Y, X, Z)\right] .
$$

\section{Nijenhuis Tensor of Connection $\bar{\nabla}$.}

The Nijenhuis tensor of semi-symmetric non-metric connection $\bar{\nabla}$ is given by

$$
\bar{N}(X, Y)=\left(\bar{\nabla}_{\bar{X}} \phi\right)(Y)-\left(\bar{\nabla}_{\bar{Y}} \phi\right)(X)-\overline{\left(\bar{\nabla}_{X} \phi\right)(Y)}+\overline{\left(\bar{\nabla}_{Y} \phi\right)(X)},
$$

where $\bar{X}=\phi X$. From equation (28), we have

$$
\left(\bar{\nabla}_{\bar{X}} \phi\right)(Y)=\left(\bar{\nabla}_{\bar{X}} \phi\right)(Y)-\eta(Y) \phi^{2} X .
$$


Interchanging $X$ and $Y$ in above equation, we get

$$
\left(\bar{\nabla}_{\bar{Y}} \phi\right)(X)=\left(\nabla_{\bar{Y}} \phi\right)(X)-\eta(X) \phi^{2} Y .
$$

Operating $\phi$ on both sides of equation (28) and using equation (3), we get

$$
\overline{\left(\bar{\nabla}_{X} \phi\right)(Y)}=\overline{\left(\bar{\nabla}_{X} \phi\right)(Y)}-\eta(Y) \phi^{2} X .
$$

Interchanging $X$ and $Y$ in above equation, we get

$$
\overline{\left(\bar{\nabla}_{Y} \phi\right)(X)}=\overline{\left(\bar{\nabla}_{Y} \phi\right)(X)}-\eta(X) \phi^{2} Y .
$$

By virtue of equations (41), (42), (43) and (44), equation (40) takes the form.

$$
\bar{N}(X, Y)=N(X, Y) \text {. }
$$

Thus, we can state as follows

Theorem 4.1 The Nijenhuis tensor of an ( $\varepsilon$ )-para Sasakian manifold is preserved under semi-symmetric non-metric connection.

In view of equation (45), we have

$$
\bar{N}(X, Y)=0 \Leftrightarrow N(X, Y)=0 .
$$

Thus, we have a theorem as follows

Theorem 4.2 An (E)-para Sasakian manifold with respect to semi-symmetric non-metric connection is integrable if and only if it is so with respect to Levi-Civita connection.

\section{Curvature Tensor of Semi-Symmetric Non-Metric Connection}

Let $\bar{R}$ be the curvature tensor of connection $\bar{\nabla}$ defined as

$$
\bar{R}(X, Y) Z=\bar{\nabla}_{X} \bar{\nabla}_{Y} Z-\bar{\nabla}_{Y} \bar{\nabla}_{X} Z-\bar{\nabla}_{[X, Y]} Z,
$$

which on using equations (8), (13) and (25), gives

$$
\begin{aligned}
& \bar{R}(X, Y) Z=R(X, Y) Z+\varepsilon[g(X, \phi Z) Y \\
& -g(Y, \phi Z) X]+\eta(Z)[\eta(Y) X-\eta(X) Y]
\end{aligned}
$$

where $\mathrm{R}(\mathrm{X}, \mathrm{Y}) \mathrm{Z}$ is curvature tensor of connection $\nabla$. From above equation, we have

$$
\begin{aligned}
& \quad \bar{R}(X, Y, Z, U)={ }^{\prime} R(X, Y, Z, U)+\varepsilon[g(X, \phi Z) g(Y, U) \\
& -g(Y, \phi Z) g(X, U)]+\eta(Z)[\eta(Y) g(X, U)-\eta(X) g(Y, U)],
\end{aligned}
$$


where ' $\bar{R}(X, Y, Z, U)=g\left({ }^{\prime} \bar{R}(X, Y) Z, U\right)$ and ' $R(X, Y, Z, U)=g(R(X, Y) Z, U)$. Let $\left\{e_{i}\right\}_{i=1}^{n}$ be an orthonormal basis of the tangent space at each point of the manifold. Putting $X=U=e_{i}$ in above equation and taking summation over $\mathrm{i}$, we get

$$
\bar{S}(Y, Z)=S(Y, Z)-(n-1) \varepsilon g(Y, \phi Z)+(n-1) \eta(Y) \eta(Z),
$$

where $\bar{S}(Y, Z)=\sum_{i=1}^{n}{ }^{\top} R\left(e_{i}, Y, Z, e_{i}\right)$ and $S(Y, Z)=\sum_{i=1}^{n}{ }^{\prime} R\left(e_{i}, Y, Z, e_{i}\right)$ are the Ricci tensors of connection $\bar{\nabla}$ and $\nabla$ respectively. Contracting above equation and using equation (10), we get

$$
\bar{r}=r+(n-1) \varepsilon,
$$

where $r=\sum_{i=1}^{n} \bar{S}\left(e_{i}, e_{i}\right)$ and $r=\sum_{i=1}^{n} S\left(e_{i}, e_{i}\right)$ are the scalar curvatures of the connections $\bar{\nabla}$ and $\nabla$ respectively.

Now, writing two more equations by the cyclic permutations of $X, Y$ and $Z$ from equation (47), we get

$$
\begin{aligned}
& \bar{R}(Y, Z,) X=R(Y, Z) X+\varepsilon[g(Y, \phi X) Z \\
& -g(Z, \phi X) Y]+\eta(X)[\eta(Z) Y-\eta(Y) Z]
\end{aligned}
$$

and

$$
\begin{aligned}
& \bar{R}(Z, X) Y=R(Z, X) Y+\varepsilon[g(Z, \phi Y) X \\
& -g(X, \phi Y) Z]+\eta(Y)[\eta(X) Z-\eta(Z) X] .
\end{aligned}
$$

Adding these two equations to equation (47) and using the fact that $R(X, Y) Z+R(Y, Z) X+$ $R(Z, X) Y=0$, we get

$$
\bar{R}(X, Y) Z+\bar{R}(Y, Z) X+\bar{R}(Z, X) Y=0
$$

Thus we have a theorem as

Theorem 5.1 An ( $\varepsilon$ )- para Sasakian manifold admitting semi-symmetric non-metric connection satisfies the condition

$$
\bar{R}(X, Y) Z+\bar{R}(Y, Z) X+\bar{R}(Z, X) Y=0 .
$$

Now, interchanging $X$ and $Y$ in equation (48), we get

$$
\begin{aligned}
& \quad \bar{R}(Y, X, Z, U)={ }^{\prime} R(Y, X, Z, U)+\varepsilon[g(Y, \phi Z) g(X, U) \\
& -g(X, \phi Z) g(Y, U)]+\eta(Z)[\eta(X) g(Y, U)-\eta(Y) g(X, U)],
\end{aligned}
$$

Adding equations (48) and (54) and using the fact that ${ }^{\prime} R(X, Y, Z, U)+{ }^{\prime} R(Y, X, Z, U)=0$, we get

$$
{ }^{\prime} \bar{R}(X, Y, Z, U)+{ }^{\prime} \bar{R}(Y, X, Z, U)=0 .
$$


Again interchanging pair of slots in equation (48), we get

$$
\begin{aligned}
& { }^{\prime} \bar{R}(Z, U, X, Y)={ }^{\prime} R(Z, U, X, Y)+\varepsilon[g(\phi Z, X) g(U, Y) \\
& -g(\phi U, X) g(Z, Y)+\eta(X)[\eta(U) g(Z, Y)-\eta(Z) g(U, Z)] .
\end{aligned}
$$

Subtracting equation (48) and (56) with the fact that ' $R(X, Y, Z, U)-{ }^{\prime} R(Z, U, X, Y)=0$, we get

$$
\begin{aligned}
& \quad \bar{R}(X, Y, Z, U)-{ }^{\prime} \bar{R}(Z, U, X, Y)=\varepsilon[g(\phi X, U) g(Y, Z) \\
& -g(\phi Y, Z) g(X, U)]+\eta(Z) \eta(Y) g(X, U)-\eta(X) \eta(U) g(Y, Z) .
\end{aligned}
$$

Thus, in view of equations (55) and (57), we can state a follows

Theorem 5.2 An ( $\varepsilon$ )-para Sasakian manifold $M^{n}$ admitting a semi-symmetric non-metric connection $\bar{\nabla}$ satisfies the condition

(i) $\bar{R}(X, Y, Z, U)+{ }^{\prime} \bar{R}(Y, X, Z, U)=0$,

(ii) ${ }^{\prime} \bar{R}(Y, X, Z, U)-{ }^{\prime} \bar{R}(Z, U, X, Y)=\varepsilon[g(\phi X, U) g(Y, Z)-g(\phi Y, Z) g(X, U)]$

$$
+\eta(Z) \eta(Y) g(X, U)-\eta(X) \eta(U) g(Y, Z) \text {. }
$$

Now, suppose $R(X, Y) Z=0$, then in view of equation (47), we have

$$
R(X, Y) Z=\varepsilon[g(Y, \phi Z) X-g(X, \phi Z) Y]+\eta(Z)[\eta(X) Y-\eta(Y) X],
$$

which gives

$$
\varepsilon \eta(R(X, Y) Z)=\varepsilon[\varepsilon g(Y, \phi Z) \eta(X)-\varepsilon g(X, \phi Z) \eta(Y)]
$$

which shows that

$$
R(X, Y) Z=\varepsilon[g(Y, \phi Z) X-g(X, \phi Z) Y]
$$

Thus, we can state as follows

Theorem 5.3 If the curvature tensor of semi-symmetric non-metric connection in an ( $\varepsilon$ )-para Sasakian manifold vanishes, then

$$
R(X, Y) Z=\varepsilon[g(Y, \phi Z) X-g(X, \phi Z) Y]
$$

\section{Projective Curvature Tensor of Semi-Symmetric Non-Metric Connection}

Projective curvature tensor $\bar{P}$ of semi-symmetric non-metric connection $\bar{\nabla}$ in an $(\varepsilon)$-para Sasakian manifold is given by

$$
\bar{P}(X, Y) Z=\bar{R}(X, Y) Z-\frac{1}{(n-1)}[\bar{S}(Y, Z) X-\bar{S}(X, Z) Y]
$$


Using equations (47) and (49) in above equation, we get

$$
\bar{P}(X, Y) Z=P(X, Y) Z,
$$

where $\mathrm{P}(\mathrm{X}, \mathrm{Y}) \mathrm{Z}$ is the projective curvature tensor of connection $\nabla$ in $M^{n}$ defined as

$$
P(X, Y) Z=R(X, Y) Z-\frac{1}{(n-1)}[S(Y, Z) X-S(X, Z) Y] .
$$

Writing two more equations by the cyclic permutations of $X, Y$ and $Z$ from equation (62) and adding them to equation (62) with the fact that $P(X, Y) Z+P(Y, Z) X+P(Z, X) Y=0$, we get

$$
\bar{P}(X, Y) Z+\bar{P}(Y, Z) X+\bar{P}(Z, X) Y=0 .
$$

Again interchanging $X$ and $Y$ in equation (62), we get

$$
\bar{P}(Y, X) Z=P(Y, X) Z
$$

Adding equations (62) and (65) with the fact that $P(X, Y) Z+P(Y, X) Z=0$, we get

$$
\bar{P}(X, Y) Z+\bar{P}(Y, X) Z=0 .
$$

Thus, in view of equations (64) and (66), we can state as follows

Theorem 6.1 An (E)-para Sasakian manifold $M^{n}$ admitting semi-symmetric non-metric connection satisfies the conditions

(i) $\bar{P}(X, Y) Z+\bar{P}(Y, Z) X+\bar{P}(Z, X) Y=0$,

(ii) $\bar{P}(X, Y) Z+\bar{P}(Y, Z) X=0$.

Now, suppose $\bar{S}=0$, then in view of equation (61), we have

$$
\bar{P}(X, Y) Z=\bar{R}(X, Y) Z .
$$

Thus by virtue of equations (65) and (67), we have

$$
\bar{R}(X, Y) Z=P(X, Y) Z .
$$

Thus, we can state as follows-

Theorem 6.2 If the Ricci tensor of semi-symmetric non-metric connection vanishes in $M^{n}$, then the curvature tensor of semi-symmetric non-metric connection is equal to the projective curvature tensor of the Levi-Civita connection in $M^{n}$. 
Again, by virtue of equations (47), (63) and (68), we have

$$
\begin{aligned}
& S(X, Z) Y-S(Y, Z) X=(n-1) \varepsilon[g(X, \phi Z) Y \\
& -g(Y, \phi Z) X]+(n-1) \eta(Z)[\eta(Y) X-\eta(X) Y] .
\end{aligned}
$$

Taking the inner product of above equation with $U$, we get

$$
\begin{aligned}
& S(X, Z) g(Y, U)-S(Y, Z) g(X, U)=(n-1) \varepsilon[g(X, \phi Z) g(Y, U) \\
& g(Y, \phi Z) g(X, U)]+(n-1) \eta(Z)[\eta(Y) g(X, U)-\eta(X) g(Y, U)] .
\end{aligned}
$$

Putting $Y=U=e_{i}$ in above equation and taking summation over $\mathrm{i}$, we get

$$
S(X, Z)=\varepsilon(n-1) g(X, \phi Z)+(n-\varepsilon) \eta(X) \eta(Z) .
$$

Thus, we have a theorem as follows-

Theorem 6.3 If the curvature tensor of semi-symmetric non-metric connection in an ( $\varepsilon$ )-para Sasakian manifold $M^{n}$ is equal to the projective curvature tensor of Levi-Civita connection, then equation (71) is satisfied.

Again, suppose $\bar{R}=0$, then in view of equation (68), we have

$$
P(X, Y) Z=0
$$

This shows that $M^{n}$ is protectively flat.

Thus, we can state as follows -

Theorem 6.4 If in $M^{n}$ the curvature tensor of semi-symmetric non-metric connection vanishes, then the manifold is projectively flat.

\section{References}

[1] Agashe, N.S. and Chafle, M.R. (1992), A semi-symmetric non-metric connection on a Riemannian manifold, Indian J. Pure Appl. Math., 23(6), 399-409.

[2] Amur K. and Pujar, S.S. (1978), On submanifolds of a Riemannian manifold admitting a semisymmetric metric connection, Tensor (N.S.), 32, 35-38.

[3] Adonie, P.O.C. (1976), On semi-symmetric non-metric connection on a Riemannian manifold, Ann. Fac. Sci. De Kinshasa, Zaire Sect. Math.-Phys., 2.

[4] Bartolotti, E. (1930), Sulla geometria dello variata a connection affine, Ann. di Math., 4(8), 53-101. 
[5] Bejancu, A. and Duggal, K.L. (1993), Real hypersurfaces of indefinite Kahler manifolds, Int. J. Math. Math.Sci., 16(3), 545-556.

[6] Chaki, M.C. and Chaki, B. (1987), On pseudo symmetric manifolds admitting a type of semisymmetric connection, Soochow J. Math., 13, 1-7.

[7] Chaki, M.C. and Konar, A. (1981), On a type of semi-symmetric connection on a Riemannian manifold, J. Pure Math., Calcutta University, 77-80.

[8] De, U.C. (1990), On a type of semi-symmetric connection on a Riemannian manifold, Indian J. Pure Appl. Math., 21(4), 334-338.

[9] De, U.C. and De, B.K. (1995), Some properties of a semi-symmetric metric connection on a Riemannian manifold, Istanbul Univ. fen Fak. Math. Der., 54, 111-117.

[10] De, U.C. and Ghosh, G.C. (1994), On a type of semi-symmetric metric connection on a Riemannian manifold, Istanbul Univ. Fen Fak. Math. Der., 53, 29-35.

[11] De, U.C. and Sarkar, A. (2009), On $\varepsilon$-Kenmotsu manifolds, Hadronic Journal, 32, 231-242.

[12] Duggal, K.L. (1990), Space time manifolds and contact structures, International Journal of Mathematics and Mathematical Sciences, 13(3), 545-553.

[13] Friedmann, A. and Schouten, J.A. (1924), Uber die geometric der halb-symmetrischen Ubertragum, Math. Zeitschr, 21, 211-233.

[14] Hayden, H.A. (1932), Subspace of space with torsion, Proc. London Math. Soc., 34, 27-50.

[15] Imai, T. (1972), Notes on semi-symmetric metric connections, Tensor (N.S.), 24, 293-296.

[16] Imai, T. (1973), Notes on semi-symmetric metric connection II, Tensor (N.S.) 27, 56-58.

[17] Mishra, R.S. and Pandey, S.N. (1978), Semi-symmetric metric connection in an almost contact manifolds, Indian J. Pure Appl. Math., 9(6), 570-580.

[18] Pravanovic, M. (1975), On pseudo metric semi-symmetric connections, Publ. De L. Institute Math., N.S., 18(32), 157-164.

[19] Sato,I. (1976): On a structure similar to the almost contact structure, Tensor (N.S.), 30(3), 219-224.

[20] Singh, R.N. and Pandey, M.K. (2008), On a type of semi-symmetric metric connection on a Riemannian manifold, Rev. Bull. Cal. Math. Soc., 16(2), 179-184.

[21] Xufeng, X. and Xiaoli, C. (1998), Two theorems on $\varepsilon$-Sasakian manifolds, Int. J. Math. Math. Sci., 21(2), 249-254.

[22] Yano, K. (1970), On semi-symmetric connection, Revue Roumanie de Mathematiques Pures et. Appliqués, 15, 1579-1581. 
S.K. Pandey, G. Pandey, K. Tiwari and R.N. Singh / J. Math. Computer Sci. 12 (2014), 159-172

[23] Yano, K. and Imai, T. (1975), On semi-symmetric metric F-connection, Tensor (N.S.), 29, 134-138.

[24] Yano, K. and Imai, T. (1982), Quarter symmetric metric connections and their curvature tensors, Tensor. (N.S.), 38, 13-18. 